\title{
Efectividad de la terapia con leuprolide acetato de depósito cada tres meses en pubertad precoz central
}

\author{
M Verónica Mericq $\mathbf{G}^{1}$, Ronald Youlton $\mathbf{R}^{1}$, \\ $M$ Eugenia Willshaw $Z^{2}$. \\ Effectiveness of Leuprolide acetate \\ therapy every three months \\ in central precocious puberty
}

Background: Precocious puberty may reduce final adult height, and affected children may suffer social and emotional problems. The efficacy of treatment with a long acting agonist analogue of the gonadotropin releasing hormone (aLHRH) has been well demonstrated. Aim: To evaluated the efficacy of a new formulation of aLHRH (leuprolide, Lupron ${ }^{\circledR}$ ) for the suppression of gonadotropin activation and clinical signs of puberty. Material and methods: Eleven children (ten females) with idiopathic central precocious puberty, with a mean chronological age of $7.5 \pm 1.8$ years and a bone age of $9.7 \pm 1.8$ years were recruited. Testicular volume in the male was 15 $\mathrm{ml}$. In females, Tanner stage for breast development was between $2-4$ and mean ovarian volume was $2.3 \pm 0.8 \mathrm{ml}$. They were treated during 18 months with $\mathrm{aLHRH}, 11.25 \mathrm{mg}$ administered intramuscularly every three months. Results: Clinical, hormonal and ultrasonographic signs of puberty regressed in all patients. The degree of suppression of $\mathrm{LH}$ was $87.7 \pm 5.1 \%$ at the end of the 18 months. No significant changes in bone mineral content were observed during the treatment period. Conclusions: Leuprolide (aLHRH) $11.25 \mathrm{mg}$, injected every three months, is effective for the control of central precocious puberty and allows to reduce the number of yearly injections from 12 to 4 (Rev Méd Chile 2006; 134: 821-6).

(Key words: Gonadotropins; Leuprolide; Puberty, precocious)

\begin{abstract}
Recibido el 12 de julio, 2005. Aceptado el 10 de enero, 2006.
${ }^{1}$ Departamento de Pediatría, Unidad de Endocrinología, Clínica Las Condes y ${ }^{2}$ Departamento de Pediatría, Hospital Militar de Santiago, Santiago, Chile.
\end{abstract}

$\mathrm{E}^{\mathrm{n}}$ el ámbito mundial, existe evidencia sobre una tendencia secular al adelanto de la edad de la pubertad, particularmente en el sexo femenino. En Chile existe información desde hace poco más de cien años, como también estudios recientes $^{1}$. Esta observación ha llevado a la Lawson Wilkins Pediatric Endocrine Society a descender los límites de edad en que se considera normal la iniciación de la pubertad en Estados Unidos de

Correspondencia a: Dra. M. Verónica Mericq G. Departamento de Pediatría, Clínica Las Condes. Lo Fontecilla 441, Las Condes, Santiago. Fax: 2-4247240. E-mail vmericq@med.uchile.cl
Norteamérica (EE.UU) ${ }^{2}$. Sin embargo, el consenso de un grupo de expertos en nuestro país, ha sugerido, por el momento, mantener el criterio de diagnóstico de pubertad precoz como aquella que se inicia antes de los ocho años en niñas y nueve años en varones. Esta decisión se ha tomado considerando que, al menos, la edad de menarquia ha sido estable en los últimos 30 años y en varones no se demostró un adelanto en la edad de inicio puberal ${ }^{3}$.

Las consecuencias de un desarrollo puberal anticipado son la pérdida de centímetros de estatura final por fusión temprana de los cartílagos de crecimiento y la eventual presentación de problemas emocionales y psicológicos. 
Actualmente existe consenso sobre la utilización de análogos agonistas superactivos del factor liberador hipotalámico de gonadotrofinas (aLHRH), en los casos de pubertad precoz central. Las base fisiológica del tratamiento deriva de las observaciones de Knobil, que demostraron que la estimulación pulsátil de la hipófisis con LHRH estimula la secreción de gonadotrofinas y que la estimulación continua la inhibe ${ }^{4}$.

Los aLHRH que se utilizan tienen una mayor afinidad por el receptor de LHRH de los gonadotrofos y una mayor resistencia a su degradación enzimáti$\mathrm{ca}^{5}$. Los objetivos de la terapia con aLHRH, en la pubertad precoz central, son la detención o regresión de los caracteres sexuales secundarios, inhibición de los ciclos menstruales, restaurar el comportamiento apropiado a la edad cronológica, restaurar el patrón de crecimiento y mejorar el pronóstico de estatura. Todos estos objetivos son razonablemente alcanzables con la terapia de aLHRH ${ }^{6,7}$.

Los primeros aLHRH sintetizados se suministraban diariamente por vía subcutánea o por vía intranasal. Posteriormente, aparecieron preparados farmacéuticos de depósito, con una duración de actividad promedio de cuatro semanas. Recientemente han aparecido formulaciones de mayor duración, que permiten administrar el medicamento en forma más alejada. Esta característica representa claramente una ventaja comparativa en términos de adherencia $y$, a veces, de costo para los pacientes.

En la actualidad, existe un único reporte sobre el uso de análogo de LHRH cada 90 días en pubertad precoz central y su efectividad en la inhibición de las gonadotrofinas durante un período de 6 meses $^{8}$.

El objetivo de este trabajo fue evaluar la eficacia de un preparado de aLHRH acetato de leuprolide (Lupron ${ }^{\circledR} 11,25 \mathrm{mg}$ ) im, cada tres meses, administrado durante un periodo de 18 meses, en la supresión de la activación gonadotrófica, signos clínicos de pubertad precoz y maduración ósea.

\section{PACIENTES Y MÉTODOS}

Once pacientes con pubertad precoz central idiopática, de acuerdo a los criterios auxológicos actualmente vigentes en nuestro país, fueron invitados a participar en el estudio. Estos pacientes fueron reclutados en el Departamento de Pediatría de Clínica Las Condes y en el Servicio de Endocrinología Infantil del Hospital Militar de Santiago. Se consideraron requisitos de inclusión la ausencia de tratamiento previo y clínicamente, el desarrollo mamario Tanner ll o mayor en niñas y un volumen testicular de 4 cc 0 más en varones. En forma adicional, debían poseer una edad ósea mayor que la edad cronológica (>1 DS para la edad cronológica), una respuesta de $\mathrm{LH}$ al estímulo de LHRH igual o superior a 5,0 UI/L ${ }^{9}$ y una concentración plasmática de testosterona superior a $1,7 \mathrm{nmol} / \mathrm{L}$ en varones. En todas las niñas se consideró, como criterio de ingreso, un volumen ovánico superior a 1,5 cc, sumados a longitud uterina superior a $36 \mathrm{~mm}$ por ecografía realizada por el mismo ecografista infantil (KM). Al momento del diagnóstico se realizó a todos los pacientes una tomografía axial computada (TAC), con contraste de cerebro y con foco hipotálamo e hipófisis, con el objeto de descartar lesiones de esa zona, que secundariamente produjeran pubertad precoz.

El protocolo de estudio fue aprobado por los Comités de Ética de ambas instituciones y, previo al inicio de la terapia, los padres firmaron un consentimiento informado.

Una vez ingresados se indicó el aLHRH acetato de leuprolide, en la formulación de 11,25 mg, que se inyectó vía im cada tres meses. Este producto fue gentilmente donado por Abbott Laboratorios de Chile.

Los pacientes asistieron a control clínico cada 3 meses, donde se realizó un examen físico completo con determinación de estatura, peso, índice de masa corporal (IMC), proporciones corporales y estadio de desarrollo puberal según Tanner ${ }^{10}$.

En forma adicional, se realizó una prueba de LHRH nativo $\left(100 \mu \mathrm{g} / \mathrm{m}^{2}\right)$ en que se determinaron los niveles plasmáticos de LH y FSH a los 0, 30 y 60 min. En cada control se realizaron, además, exámenes de orina completa, perfil bioquímico y hemograma.

Se consideró que la frenación de gonadotrofinas fue adecuada cuando la concentración máxima de $L H$, post estímulo, alcanzó un valor inferior a inferior a 3,0 UI/L, la de testosterona menor a 1 $\mathrm{nmol} / \mathrm{L}$ (varones) y la de estradiol fue inferior a 73 $\mathrm{pmol} / \mathrm{L}$ (mujeres) ${ }^{9}$. La duración del estudio fue de 18 meses (6 inyecciones).

Cada 6 meses se determinó la edad ósea en todos los pacientes mediante radiografía de la mano, utilizando el método de Greulich y Pyle (informe, KM, Clínica Las Condes). Al mismo tiempo, se realizó control ecográfico en cada una de las niñas participantes. La ecografía ginecológica fue realizada siem- 
pre por el mismo radiólogo (KM, Clínica Las Condes), utilizando un equipo ATL HDI 5000 Phillips.

Al inicio y en el control final de los 18 meses se realizó una densitometría ósea de columna lumbar, utilizando un densitómetro marca Lunar modelo DPX alpha. El informe entrega los datos como contenido mineral óseo y porcentaje de adecuación para la densidad mineral areal, ambos en columna lumbar.

Se consideraron criterios de suspensión lograr una predicción estatural cercana ( \pm 1 desviación estándar) al pronóstico familiar y evitar la progresión del desarmollo de caracteres sexuales antes de lo considerado normal, para evitar los problemas conductuales y sicológicos que se pueden observar en estas condiciones. En forma adicional también se consideró criterio de exclusión el presentar reacciones adversas locales en el sitio de la administración del análogo o en los exámenes de laboratorio general.

Determinaciones hormonales. Todas las mediciones de LH, FSH, testosterona y estradiol fueron realizadas por electroquimioluminiscencia (ECLA) en el Laboratorio de Hormonas de Clínica Las Condes. Estas determinaciones tienen un coeficiente de variación intraensayo entre 4 y $6 \%$ e interensayo entre 10 y $12 \%$. La sensibilidad del método para $\mathrm{LH}$ fue de $0,1 \mathrm{UI} / \mathrm{L}$, para FSH de 0,1 UI/l, para testosterona de 0,069 $\mathrm{nmol} / \mathrm{L}$ y para estradiol de 18 $\mathrm{pmol} / \mathrm{L}$ con esta metodología.

Estadística. Los resultados se muestran como promedio \pm desviación estándar (DE). El análisis estadístico se realizó con el programa SPSS 10.0 para Windows. Utilizando una $t$ de student cuando los datos se distribuyeron en forma normal y test de Wilcoxon cuando su distribución fue no paramétrica.

\section{RESUlTADOS}

Ingresaron al estudio 11 pacientes (10 mujeres, 1 varón) con una edad cronológica promedio de $7,5 \pm 1,8$ años y edad ósea promedio de $9,7 \pm 1,8$ años.

En las 10 niñas, el tratamiento se inició antes de los 9 años de edad. En el varón se inició a los 11 años. El lapso de tiempo promedio transcurrido entre los primeros síntomas y el inicio de la terapia fue 1,5 $\pm 0,5$ años.

El desarrollo mamario en las niñas estaba en estadios 2 a 3 de Tanner. El único paciente de sexo masculino presentaba un volumen testicular de 15 cc bilateral.

En la Tabla 1 se detallan los parámetros clínicos (edad cronológica, género, estatura inicial y final en desviaciones estándar, peso [kgl IMC [percentiles para edad y sexol, velocidad de crecimiento) para cada uno de los pacientes ingresados al estudio, al ingreso (I) y al final (F) de 18 meses de terapia con aLHRH.

En el primer control clínico, a los tres meses de inyectada la primera dosis de acetato de leuprolide, se observó regresión de los caracteres secundarios en todos los pacientes, con excepción del vello genital. Las glándulas mamarias redujeron su consis-

Tabla 1. Parámetros clínicos al ingreso (I) y al final (F) de 18 meses de terapia con aLH RH

\begin{tabular}{|cccccccccc|}
\hline $\begin{array}{c}\text { Paciente } \\
\text { No }\end{array}$ & $\begin{array}{c}\text { EC } \\
\text { (años) }\end{array}$ & $\begin{array}{c}\text { Género } \\
\text { (M/V) }\end{array}$ & $\begin{array}{c}\text { Estatura I } \\
\text { (DE) }\end{array}$ & $\begin{array}{c}\text { Estatura F } \\
\text { (DE) }\end{array}$ & $\begin{array}{c}\text { Peso I } \\
(\mathrm{kg})\end{array}$ & $\begin{array}{c}\text { Peso F } \\
(\mathrm{kg})\end{array}$ & $\begin{array}{c}\text { IMC I } \\
\text { (percentil) }\end{array}$ & $\begin{array}{c}\text { IMC F } \\
\text { (percentil) }\end{array}$ & $\begin{array}{c}\text { Vel.crec. } \\
\text { (cm/año) }\end{array}$ \\
\hline 1 & 6,7 & $\mathrm{M}$ & 2,1 & 2,0 & 30,5 & 35,6 & 89,0 & 83,0 & 6,7 \\
2 & 11,7 & $\mathrm{~V}$ & $-1,0$ & $-1,4$ & 41,0 & 44,1 & 93,0 & 76,0 & 4,7 \\
3 & 8,8 & $\mathrm{M}$ & 0,8 & 0,4 & 35,5 & 44,3 & 87,0 & 93,0 & 4,5 \\
4 & 8,0 & $\mathrm{M}$ & 1,4 & 1,5 & 30,0 & 40,3 & 60,0 & 85,0 & 6,7 \\
5 & 7,0 & $\mathrm{M}$ & 0,5 & 0,7 & 30,0 & 34,0 & 96,0 & 95,0 & 4,7 \\
6 & 8,5 & $\mathrm{M}$ & 2,3 & 1,0 & 36,8 & 42,5 & 80,0 & 76,0 & 5,3 \\
7 & 4,8 & $\mathrm{M}$ & 2,4 & 1,8 & 22,5 & 26,0 & 67,0 & 69,0 & 5,3 \\
8 & 8,0 & $\mathrm{M}$ & 3,0 & 2,9 & 39,7 & 56,0 & 92,0 & 99,0 & 6,0 \\
9 & 7,4 & $\mathrm{M}$ & 1,5 & 1,6 & 26,5 & 33,0 & 15,2 & 49,0 & 6,7 \\
10 & 5,5 & $\mathrm{M}$ & $-0,3$ & 0,1 & 23,7 & 29,0 & 97,6 & 97,9 & 6,0 \\
11 & 6,6 & $\mathrm{M}$ & $-1,4$ & & 19,4 & & 49,0 & & 0,0 \\
Promedio & 7,5 & & 0,9 & 1,1 & 30,5 & 38,5 & 75,1 & 82,3 & 5,1 \\
DE & 1,8 & & 1,4 & 1,2 & 7,1 & 8,8 & 25,4 & 15,5 & 1,9 \\
\hline
\end{tabular}

Se detalla edad cronológica, género, estatura inicial y final en desviaciones estándar (DE), peso $(\mathrm{kg})$, índice de masa corporal (IMC) en percentiles para edad y sexo y velocidad de crecimiento en los 18 meses ajustada a 12 meses. 
tencia y su diámetro en algunos casos hasta límites no precisables (al ingreso $7,7 \pm 8,2 \mathrm{~cm}$ versus $4,9 \pm 3,7$ $\mathrm{cm}, \mathrm{p}<0,05$ ), como también el aspecto de la mucosa vaginal volvió a características infantiles. Esto último no pudo ser cuantificado, pues no se hizo estudio y seguimiento de la citología vaginal. Así mismo, el volumen testicular del varón se redujo de 15 a 12 cc a los seis meses de tratamiento.

La supresión gonadotrófica se demostró a través de la concentración máxima de $\mathrm{LH}$ al estímulo de LHRH en el control del sexto mes que se encontró en niveles prepuberales en todos $(\mathrm{LH}$ $2,05 \pm 1,1 \mathrm{UI} / \mathrm{L}$ ), excepto una paciente, en que este hecho ocurrió en el control siguiente. Tanto las concentraciones de estradiol en las niñas como la de testosterona en el varón disminuyeron respecto de los valores basales (estradiol basal 73,9 $\pm 48,3$ $\mathrm{pmol} / \mathrm{L}$ versus $44 \pm 15$ a los 3 meses y $47,7 \pm 33$ a los 18 meses y testosterona $8 \mathrm{nmol} / \mathrm{l}$ versus $0,5 \mathrm{nmol} / \mathrm{L}$ a los 3 meses y 0,2 nmol/L a los 18 meses).

Los genitales internos también mostraron una regresión rápida. Al sexto mes el volumen ovárico inicial de 2,3 $\pm 0,8 \mathrm{cc}$, se redujo a $1,9 \pm 0,7 \mathrm{cc}$, diferencia que no alcanzó a ser significativa estadísticamente. La longitud uterina, sin embargo, se redujo de $4,3 \pm 1,0$ a $3,9 \pm 0,9 \mathrm{~cm}(\mathrm{p}<0,05)$.

En el seguimiento a más largo plazo un parámetro importante de evaluar es la velocidad de madura- ción ósea. La edad ósea, inicialmente avanzada, mostró una desaceleración al final del período de estudio. Esto se demuestra a través del delta de edad ósea - edad cronológica (EO-EC) que se redujo de $2,2 \pm 1,1$ a $1,7 \pm 1,0$ años al final del período de 18 meses de terapia ( $p<0,01$ ) lo que mejoró el pronóstico de estatura inicial. Sin embargo, este último parámetro es de poca utilidad con una terapia de 18 meses por lo que estos datos no se muestran.

En el aspecto antropométrico, la estatura promedio inicial expresada en desviaciones estándar (DE) para la edad y sexo en los 11 pacientes fue de $0,9 \pm 1,4 \mathrm{DS}$. Al cabo de los 18 meses de tratamiento este puntaje cambió a 1,1 $\pm 1,2$ DS lo que no es una diferencia significativa. Así también, el índice de masa corporal inicial (IMC) promedio estaba en el percentil $75 \pm 25 \mathrm{y}$ al final del tratamiento estaba en el percentil 82,3 $\pm 15,5$ ( $p=N S$ ). La información sobre los parámetros de laboratorio al ingreso y a los 18 meses de tratamiento con aLHRH se resumen en la Tabla 2.

En algunas niñas, después de la primera inyección hubo un pequeño efecto estimulatorio inicial, pero ninguna presentó sangrado vaginal. Ningún paciente presentó reacciones locales en el sitio de la inyección ni se observaron efectos adversos durante el curso del tratamiento atribuibles a éste.

Los pacientes mostraron buena adherencia al tratamiento. Sólo una niña no concurrió al último

Tabla 2. Evolución de los parámetros de laboratorio al inicio (I) y final (F) de los 18 meses de terapia con Lupron ${ }^{\circledR} 11,25 \mathrm{mg} \mathrm{c} / 3$ meses

\begin{tabular}{|crrrrrrrrrr|}
\hline $\begin{array}{c}\text { Paciente } \\
\text { No }\end{array}$ & $\begin{array}{r}\text { EO I } \\
\text { años }\end{array}$ & $\begin{array}{r}\text { EO F } \\
\text { años }\end{array}$ & $\begin{array}{r}\text { LH I } \\
\text { UI/L }\end{array}$ & $\begin{array}{c}\text { LH F } \\
\text { UI/L }\end{array}$ & $\begin{array}{c}\text { Ov I } \\
\text { cc }\end{array}$ & $\begin{array}{c}\text { Ov F } \\
\text { cc }\end{array}$ & $\begin{array}{r}\text { Utero I } \\
\text { cm }\end{array}$ & $\begin{array}{r}\text { Utero F } \\
\text { cm }\end{array}$ & $\begin{array}{r}\text { DMO I } \\
(\%)\end{array}$ & $\begin{array}{r}\text { DMO F } \\
(\%)\end{array}$ \\
\hline 1 & 10,0 & 10,5 & 7,0 & 1,0 & 1,6 & 0,8 & 4,7 & 1,5 & 111,0 & 100,0 \\
2 & 12,5 & 12,8 & 20,0 & 3,1 & & & & & 102,0 & 106,0 \\
3 & 12,0 & 12,5 & 5,6 & 1,0 & 2,6 & 1,3 & 5,6 & 1,5 & 107,0 & 103,0 \\
4 & 11,0 & 12,0 & 18,8 & 2,8 & 2,8 & 1,4 & 4,4 & 4,8 & 96,0 & 99,0 \\
5 & 7,6 & 8,6 & 23,9 & 1,7 & 1,6 & 0,8 & 3,4 & 3,2 & 118,0 & 116,0 \\
6 & 11,0 & 12,0 & 21,0 & 0,9 & 2,7 & 1,3 & 3,2 & 4,1 & 87,0 & 93,0 \\
7 & 8,0 & 9,0 & 21,6 & 3,0 & 2,9 & 1,4 & 4,0 & 3,7 & 114,0 & 114,0 \\
8 & 11,0 & 12,0 & 12,0 & 1,7 & 2,6 & 1,3 & 6,0 & 4,0 & 110,00 & 113,0 \\
9 & 8,7 & 10,5 & 12,3 & 0,5 & 1,7 & 0,9 & 3,2 & 3,7 & 109,0 & 111,0 \\
10 & 8,0 & 8,5 & 12,0 & 2,0 & 0,9 & 0,4 & 3,7 & 3,6 & 112,0 & 111,0 \\
11 & 7,3 & ND & 2,8 & & 3,8 & & 4,7 & & 102,0 & \\
Promedio & 9,7 & 10,8 & 14,3 & 1,8 & 2,3 & 1,1 & 4,3 & 3,3 & 106,2 & 106,6 \\
DE & 1,8 & 1,7 & 7,2 & 0,9 & 0,9 & 0,4 & 1,0 & 1,1 & 8,9 & 7,6 \\
\hline
\end{tabular}

Se presentan los datos correspondientes a edad ósea (años), valor máximo de LH obtenido en la prueba de LHRH (UI/L), volumen ovárico $(\mathrm{cc})$, diámetro longitudinal del útero $(\mathrm{cm})$ y datos del porcentaje de adecuación para la densidad mineral ósea areal en columna lumbar ajustados para la edad y sexo. 
control por cambio de residencia a un lugar alejado de Santiago.

$\mathrm{Al}$ momento de redactar esta comunicación, 10 pacientes acuden a control regular. Dos continúan el uso del análogo (pacientes $\mathrm{n}$ - 5 y 10), un paciente completó 3 años de terapia y tres completaron 2 años de terapia. En los 8 pacientes que ya suspendieron el aLHRH se ha documentado la reactivación del eje gonadotropo.

En el paciente varón, esto se ha documentado con testosteronas matinales (último valor, 15,6 $\mathrm{nmol} / \mathrm{L})$ y aumento de volumen testicular a $25 \mathrm{cc}$ al año posterior a suspensión del análogo. En las niñas $(n=7)$ que han suspendido el análogo, la menarquia ha ocurrido en 3 de 7 de ellas y este evento ocurrió en un lapso entre 6 y 18 meses post suspensión del aLHRH. En las niñas restantes se ha observado un aumento progresivo del estadio de Tanner mamario así como cambios estrogénicos en la mucosa genital.

\section{DisCUSIÓN}

En esta expeniencia se demuestra que el uso de acetato de leuprolide de depósito, en dosis de 11,25 mg, inyectado intramuscular cada tres meses es efectivo en el tratamiento de la pubertad precoz central.

Clínicamente todos los pacientes mostraron regresión de sus signos de pubertad, lo que se acompañó de una caída en la velocidad de crecimiento a niveles prepuberales y de una leve elevación del IMC.

Las gonadotrofinas hipofisiarias fueron efectivamente frenadas en $85,6 \pm 6,9 \%$ de los valores a los tres meses de tratamiento y en $87,7 \pm 5,1 \%$ a los 18 meses. Paralelamente, tanto los niveles de estradiol, en las mujeres, como testosterona, en el varón, cayeron a niveles prepuberales durante el curso del estudio. Es importante destacar que las fluctuaciones de los niveles diarios de estradiol hacen que este examen sea poco confiable como parámetro de control de frenación de la actividad del eje gonadotropogonadal, por lo que es útil acompañar la evaluación de otros índices como el test de LHRH nativo y la ecografía pélvica. En los varones, sin embargo, los niveles de testosterona son muy útiles, como lo demostró nuestro único caso de sexo masculino, cuya concentración plasmática de testosterona inicial de $8,0 \mathrm{nmol} / \mathrm{L}$ se mantuvo bajó $0,2 \mathrm{nmol} / \mathrm{L}$ durante el tratamiento. En el estudio de $\mathrm{Carel}^{8}$, donde se utilizaron dosis similares a las nuestras, se observó una pobre supresión de los niveles de testosterona al primer mes, posterior a la primera dosis de aLHRH. El descenso se hizo evidente al tercer mes y durante el seguimiento. Esta información contrasta con lo reportado en adultos con cáncer de próstata tratados con 11,25 mg im, cada tres meses, en que se ha comunicado que las concentraciones de testosterona ya están adecuadamente suprimidas a las cuatro semanas de terapia ${ }^{11,12}$.

En concordancia con la frenación de gonadotrofinas y de esteroides sexuales, se demostró disminución del volumen ovárico y de la longitud del útero, medidas ecográficamente. El volumen testicular de nuestro paciente de sexo masculino también se redujo.

Todo lo anterior se correlaciona bien con la desaceleración de la velocidad de maduración esquelética, que se confirmó en todos los pacientes. En sólo 18 meses de observación esta desaceleración fue significativa; en un periodo más prolongado ésta probablemente sería mayor, lo cual hace que el cálculo de proyección de estatura final no sólo interrumpa su deterioro sino que mejore, al menos parcialmente.

La densidad mineral ósea tampoco se vio afectada por el tratamiento, hecho que ha sido comunicado en otras publicaciones ${ }^{13}$. Sin embargo, este es un aspecto que en terapias más prolongadas podría alterarse y en las cuales incluso se ha sugerido la utilización de calcio suplementario ${ }^{14}$.

Estos datos nos sugieren que la dosis utilizada en este estudio fue adecuada para nuestros propósitos. Existe controversia en la literatura respecto de cuál sería la más apropiada para las formulaciones de aLHRH de 3,75 mg para uso mensual. Publicaciones europeas señalan que $120 \mu \mathrm{g} / \mathrm{kg}$ de peso cada cuatro semanas, es decir 3,75 mg mensuales senían, en general, suficientes para suprimir las gonadotrofinas en niños con menos de $30 \mathrm{~kg}$ de peso y pubertad precoz central ${ }^{8}$. Esta cantidad contrasta con las recomendaciones norteamericanas de 300 $\mu \mathrm{g} / \mathrm{kg}$ cada cuatro semanas ${ }^{15-17}$ y en el otro extremo las dosis que en Japón se utilizan. En este último país, Tanaka ha demostrado que la dosis mínima efectiva es de $30 \mu \mathrm{g} / \mathrm{kg}^{18}$.

Las reacciones locales después de inyectar aL$\mathrm{HRH}$ (dolor prolongado, formación de granulomas o de un absceso estéril) ocurren en 3 a 13\% de los pacientes. En ninguno de los pacientes participantes 
en este estudio observamos tales situaciones, como tampoco presentaron otros efectos adversos ni modificaciones en el hemograma, perfil bioquímico o en el examen de orina, lo que permitió una buena adherencia hasta completar el estudio.

En conclusión, el uso de acetato de leuprolide $11,25 \mathrm{mg}$ inyectado cada tres meses ha demostra-

\section{REFERENCIAS}

1. Codner E, Unanue N, Gaete X, Barrera A, MookKanamori D, BazÁes R et al. [Age of pubertal events in Chilean school age girls and its relationship with socioeconomic status and body mass index]. Rev Méd Chil 2004; 132: 801-8.

2. KaPLowitz PB, OBERFIELD SE. Reexamination of the age limit for defining when puberty is precocious in girs in the United States: implications for evaluation and treatment. Drug and Therapeutics and Executive Committees of the Lawson Wilkins Pediatric Endocrine Society. Pediatrics 1999; 104: 936-41.

3. García H, Youlton R, Burrows R, Catani A. [Consensus on the diagnosis and treatment of central early puberty]. Rev Méd Chile 2003; 131: 95-110.

4. WiLd L, Marshall G, KNobil E. Experimental induction of puberty in the infantile female rhesus monkey. Science 1980; 207: 1373-5.

5. ОкаDA H. One- and three-month release injectable microspheres of the LH-RH superagonist leuprorelin acetate. Adv Drug Deliv Rev 1997; 28: 43-70.

6. Crowiey WF JR, Comite F, Vaie W, Rvier J, Loriaux DL, CUTLER GB JR. Therapeutic use of pituitary desensitization with a long-acting LHRH agonist: a potential new treatment for idiopathic precocious puberty. J Clin Endocrinol Metab 1981; 52: 370-2.

7. Kiein KO, Barnes KM, Jones JM, Feuilan PP, Cutler GB JR. Increased final height in precocious puberty after long-term treatment with LHRH agonists: the National Institutes of Health experience. J Clin Endocrinol Metab 2001; 86: 4711-6.

8. Carel JC, lahlou N, Jaramilo O, Montauban V, Teinturier C, Cole et al. Treatment of central precocious puberty by subcutaneous injections of leuprorelin 3-month depot (11.25 mg). J Clin Endocrinol Metab 2002; 87: 4111-6.

9. Neely EK, Hintz RL, Wilson DM, Lee PA, Gautier T, ARgENTE J ET AL. Normal ranges for immunochemiluminometric gonadotropin assays. J Pediatr 1995; 127: 40-6. do ser efectivo y seguro para la disminución de los signos clínicos, frenación de gonadotrofinas y avance de la maduración ósea en niños con pubertad precoz central. El hecho de disponer de un preparado de liberación lenta que permita ser utilizado cada tres meses favorece la adherencia a una terapia que es prolongada.

10. MARShaL WA, TANNER JM. Variations in pattern of pubertal changes in girls. Arch Dis Child 1969; 44: 291-303.

11. KHAN MS, O'BRIEN A. An evaluation of pharmacokinetics and pharmacodynamics of leuprorelin acetate 3M-depot in patients with advanced and metastatic carcinoma of the prostate. Urol Int 1998; 60: 33-40.

12. Wechsel HW, Zerbib M, Pagano F, Coptcoat MJ. Randomized open labelled comparative study of the efficacy, safety and tolerability of leuprorelin acetate $1 \mathrm{M}$ and $3 \mathrm{M}$ depot in patients with advanced prostatic cancer. Eur Urol 1996; 30 Suppl 1: 7-14; discussion 19-21.

13. NeELY EK, Bachrach LK, Hintz RL, HabiBy RL, Stemenda CW, Feezle L et al. Bone mineral density during treatment of central precocious puberty. J Pediatr 1995; 127: 819-22.

14. Antoniazzi F, Zamboni G, Bertoldo F, Lauriola S, Mengarda F, Pietrobeu A et al. Bone mass at final height in precocious puberty after gonadotropinreleasing hormone agonist with and without calcium supplementation. J Clin Endocrinol Metab 2003; 88: 1096-101.

15. Clemons RD, Kappy MS, Stuart TE, Perelman AH, HoEKSTRA FT. Long-term effectiveness of depot gonadotropin-releasing hormone analogue in the treatment of children with central precocious puberty. Am J Dis Child 1993; 147: 653-7.

16. Kaplan SL, Grumbach MM. Clinical review 14: Pathophysiology and treatment of sexual precocity. J Clin Endocrinol Metab 1990; 71: 785-9.

17. Parker KL, Baens-Bailon RG, Lee PA. Depot leuprolide acetate dosage for sexual precocity. J Clin Endocrinol Metab 1991; 73: 50-2.

18. Tanaka T, Hibi I, Kato K, Saito S, Shimizu N, Suna S et AL. A dose finding study of a super long-acting luteinizing hormone-releasing hormone analog (leuprolide acetate depot, TAP-144-SR) in the treatment of central precocious puberty. The TAP-144SR CPP Study Group. Endocrinol Jpn 1991; 38: 369-76. 